\title{
Infarto agudo al miocardio en pacientes de 80 y más años. Evolución hospitalaria y seguimiento
}

\author{
Héctor U galde, Pilar Espinosa a, G onzalo Pizarroa, \\ Ximena Dreysea. \\ Clinical features and prognosis \\ of acute myocardial infarction among \\ patients aged 80 years or older
}

Background: Subjects older than 80 years have more complicated acute myocardial infarctions and are subjected to less reperfusion procedures. Aim: To describe the hospital evolution and follow up of patients older than 80 years, suffering an acute myocardial infarction. Material and methods: Patients older than 80 years, were selected from a prospective registry of acute myocardial infarction. Among these, the hospital evolution, mortality and events during a period of five years of follow up, were recorded. Results: Of a total of 1200 patients, 83 (7\%) were aged 80 years or older. Among these, 59\% were male and 22\% were diabetic. The mean lapse between onset of symptoms and admission was 11 hours and 59\% were admitted with less than 6 hours of evolution. Sixty three percent had a history of a previous infarction and $24 \%$ had a Killip IV classification on admission. Reperfusion therapy was done in $19 \%$ and $70 \%$ had heart failure during evolution. A coronary angiography was done in $22 \%$ and $6 \%$ were subjected to revascularization. Mortality was $34 \%$, specially due to cardiogenic shock or ventricular rupture. Of 55 patients discharged from the hospital, 31\% died within one year and $64 \%$ within five years, mainly due to cardiac causes. Conclusions: Patients aged 80 years or older have more complicated myocardial infarctions, most of them are managed conservatively and long term mortality is high (Rev Méd Chile 2008; 136: 694-700).

(Key w ords: Aged, 80 and older; Myocardial infarction; Reperfusion)

Recibido el 2 de agosto, 2007. Aceptado el 6 de marzo, 2008.

Centro Cardiovascular, Hospital Clínico Universidad de Chile. Santiago de Chile.

anterno Medicina, Universidad de Chile

$E^{n}$ los últimos años la población de los países desarrollados ha experimentado grandes cambios, con tendencia al aumento de la población mayor de 65 años. En Chile este grupo ha aumentado de manera significativa alcanzando $8 \%$

Correspondencia a: Dr. Héctor Ugalde P. Santos Dumont 999, Independencia, Santiago. Fono: 9788356. E mail: hugalde@redclinicauchile.cl en $2002^{1}$. Dentro de ellos, los mayores de 80 años alcanzaban $1,6 \%$ del total y se espera un aumento sustancial para 2025, cuando alcanzarían a 2,9\% y en 2050 alrededor de $7 \%$ de la población ${ }^{2}$. Los adultos mayores (AM) constituyen un grupo mayoritario en la enfermedad cardiovascular, siendo el $60 \%$ de los pacientes hospitalizados por infarto agudo al miocardio y el $80 \%$ por insuficiencia cardíaca en Estados Unidos de Norteamérica ${ }^{3}$. 
El infarto agudo al miocardio (IAM) presenta características especiales en los AM. Se observa una alta tasa de infartos silentes (22\%-68\%), que los hace más susceptibles a eventos coronarios posteriores ${ }^{4}$, junto a esto se observa un importante retardo en la consulta por IAM ${ }^{5}$. Además, los pacientes con la edad aumentan su comorbilidad y varía la distribución de sexos, aumentando la representación femenina ${ }^{6-8}$ y en ellas se evidencia una mayor incidencia de complicaciones mortales ${ }^{9,10}$.

Según las estadísticas internacionales, las enfermedades cardiovasculares son la principal causa de muerte en todos los grupos etarios, siendo mayor la representación a mayor edad ${ }^{11}$. En Chile, la mortalidad por enfermedades cardiovasculares es cercana a $27 \%$ del total, siendo el IAM responsable de alrededor de un tercio de ellas ${ }^{12}$. El análisis de la mortalidad por IAM desde 1990 a 2001 muestra una disminución de la tasa de mortalidad (TM) ajustada, siendo actualmente de 51/100.000 hab. Además, se aprecia que la TM aumenta exponencialmente con la edad, siendo el punto de mayor mortalidad en los mayores de 80 años $^{12}$.

A lo lango de los años, se han implementado nuevas terapias para el tratamiento del IAM: trombolisis, angioplastia y by-pass coronario. Se ha probado la real utilidad de estas terapias en $\mathrm{AM}^{13-15}$, especialmente en el grupo de octogenarios $^{13}$. Pese a esto, se ha visto una importante subutilización de éstas, así como también un retardo en el tratamiento de reperfusión en los $\mathrm{AM}^{10,16}$. Berger et $\mathrm{al}^{16}$ encontraron factores asociados al retardo en la reperfusión como la vejez, diabetes, sexo femenino, historia de insuficiencia cardíaca, HTA y una elevación del segmento ST menor, todos factores más prevalentes en el grupo de $\mathrm{AM}^{16}$. Por otro lado, se ha visto que este grupo presenta más factores de riesgo para complicaciones de las terapias de reperfusión; sin embargo, Stenestrad et $\mathrm{al}^{17}$, en un estudio prospectivo, observaron una disminución de la mortalidad al año con el uso de trombolisis ${ }^{17}$. Así también, Graham et $\mathrm{al}^{18}$ mostraron que la terapia intervencionista precoz (angioplastia, cirugía) mejora la sobrevida en los AM, encontrándose una mayor reducción del riesgo en los mayores de 80 años comparado con los más jóvenes ${ }^{18}$.

Es así como nos enfrentamos a un nuevo grupo de pacientes, los octogenarios, con características particulares, cuyo número de individuos va en aumento. Como hemos visto, existen estudios extranjeros que se han dedicado a ellos, sin embargo, en Chile es escasa la información disponible sobre el tema. En vista de los cambios demográficos que hemos experimentado en los últimos años y los que se esperan a futuro, se hace fundamental describir y estudiar las características de este grupo etario.

\section{OBjetivos}

Describir las características clínicas, evolución hospitalaria y el seguimiento en cuanto a mortalidad al año y hasta 5 años en pacientes de 80 años y más que ingresan con diagnóstico de IAM.

\section{Material y MÉTOdo}

De un registro prospectivo consecutivo de 1.200 pacientes, ingresados con diagnóstico de IAM a nuestro Centro Cardiovascular del Hospital Clínico de la Universidad de Chile desde el 1 de enero de 1988 hasta el 31 de diciembre de 2001, se seleccionó a todos los pacientes de 80 y más años. Para el diagnóstico de IAM se utilizó los mismos criterios aplicados por el grupo GEM ${ }^{19}$, basados en la clínica, cambios del electrocardiograma y ascenso de enzimas miocárdicas.

En ellos se revisó su historia clínica, registrándose sus características demográficas, historia cardiovascular previa, morbilidad asociada, tipo de IAM, tiempo de evolución al ingreso, situación hemodinámica inicial, tratamiento inicial, evolución hospitalaria, exámenes complementarios, tratamiento en la hospitalización, días de hospitalización, tratamiento al alta y seguimiento en cuanto a mortalidad al año y cinco años, sea por revisión de su historia o solicitud en la Oficina de Registro Civil de antecedentes de defunción.

\section{RESULTADOS}

De los 1.200 pacientes con IAM, 83 tenían 80 o más años (7\%) (Tabla 1), con un promedio de edad de 83,5 años, 59\% eran de sexo masculino. Los factores de riesgo cardiovascular más frecuentes fueron hipertensión arterial (HTA) y diabetes mellitus (DM). 
Tabla 1. Características de la población en estudio

\begin{tabular}{|lcr|}
\hline Serie & $\mathrm{n}$ & $\%$ \\
\hline Pacientes & 83 & \\
Hombres & 49 & $59 \%$ \\
Mujeres & 34 & $41 \%$ \\
Edad & $83,5 \pm 3,0(80-93)$ & \\
Factores de riesgo & & \\
Tabaquismo & 9 & $11 \%$ \\
HTA & 49 & $59 \%$ \\
DM & 18 & $22 \%$ \\
Dislipidemias & 5 & $6 \%$ \\
Obesidad & 3 & $4 \%$ \\
Antecedentes previos & & \\
Angina & 35 & $42 \%$ \\
Estable & 15 & $43 \%$ \\
Inestable & 20 & $57 \%$ \\
IAM & 11 & $13 \%$ \\
Cirugía & 1 & $1 \%$ \\
Hora de consulta & & \\
Promedio & $11,2 \pm 14,0(1-72)$ & \\
Antes 6 h & 49 & $59 \%$ \\
Antes 12 h & 65 & $78 \%$ \\
\hline
\end{tabular}

Tabla 2. Variables hemodinámicas al ingreso

\begin{tabular}{|lrr|}
\hline & Promedio & \multicolumn{1}{c|}{ Rango } \\
\hline Frecuencia cardíaca & $84,5 \pm 24,9$ & $40-150$ \\
PAS, mmHg & $123 \pm 27$ & $50-170$ \\
Killip ingreso & $1,9 \pm 1,1$ & $1-4$ \\
Killip máximo & $2,3 \pm 1,2$ & $1-4$ \\
Killip IV & 20 P (24\%) & \\
\hline
\end{tabular}

Tabla 3. C aracterísticas del IAM

\begin{tabular}{|lrr|}
\hline & $\mathrm{N}$ & $\%$ \\
\hline ECG: & & \\
Supradesnivel ST & 59 & 71,1 \\
Infradesnivel ST & 15 & 18,1 \\
BCRI & 3 & 3,6 \\
Sin alteraciones & 6 & 7,2 \\
Localización: & & \\
Anterior & 52 & 62,7 \\
Inferior & 21 & 25,3 \\
Inferolateral & 7 & 8,4 \\
Posterior & 3 & 3,6 \\
\hline
\end{tabular}

En relación a historia previa, 56,6\% tenía antecedentes cardiovasculares previos, siendo la presencia de angina el antecedente más común, seguida por el infarto al miocardio, sólo 1 paciente había sido intervenido quirúrgicamente.

Con respecto a las características del evento, se observó que el tiempo desde que se iniciaron los síntomas a la consulta fue en promedio de 11,2 h, $78 \%$ ingresó antes de las $12 \mathrm{~h}$ de evolución del IAM. El estado hemodinámico al ingreso de los pacientes se puede observar en la Tabla 2. Cabe señalar que $24 \%$ de los pacientes ingresaron en Killip 4.

En el electrocardiograma (ECG) de ingreso (Tabla 3) predominó el supradesnivel del ST (SDST) con $71,1 \%$, con un bajo porcentaje de infradesnivel del ST (IDST) y otras alteraciones.

El IAM se localizó principalmente en pared anterior, $62,7 \%$, y en menor cuantía en pared inferior. La localización del IAM se definió en base al ECG de ingreso y al ecocardiograma.

El máximo valor de las enzimas fue de $1.180 \pm 1.201$ para CK y $126 \pm 141$ para $\mathrm{CKmb}$, en promedio a las $21,4 \pm 6,1 \mathrm{~h}(6-30)$.

El 97,6\% recibió aspirina como tratamiento farmacológico inicial, 65\% nitroglicerina endovenosa, 33,7\% heparina, 10,8\% betabloqueadores y $7 \%$ inhibidores de enzima de conversión. El 19,2\% de los pacientes recibió terapia de reperfusión, de éstos 10,8\% trombolisis y $8,4 \%$ fue tratado con angioplastia primaria.

En la evolución hospitalaria, 57 pacientes $(68,6 \%)$ presentaron complicaciones, lo que podemos ver en la Tabla 4. Las principales fueron la

Tabla 4. Complicaciones intrahospitalarias

\begin{tabular}{|lrr|}
\hline & $\mathrm{N}$ & $\%$ \\
\hline Insuficiencia cardíaca & 56 & $67 \%$ \\
Shock & 21 & $25 \%$ \\
BAV 2-3 grado & 19 & $23 \%$ \\
BAV completo & 15 & $18 \%$ \\
AV malignas & 8 & $10 \%$ \\
FV & 5 & $6 \%$ \\
TVS & 3 & $4 \%$ \\
Fibrilación auricular & 8 & $10 \%$ \\
Angina post IAM & 6 & $7 \%$ \\
Rotura ventricular & 3 & $4 \%$ \\
CIV & 1 & $1 \%$ \\
IM aguda & 1 & $1 \%$ \\
\hline
\end{tabular}


insuficiencia cardíaca, seguida por bloqueo aurículo-ventricular (BAV) de alto grado y arritmias ventriculares malignas.

Durante la estadía hospitalaria se complementó el estudio con ecocardiografía en $78,3 \%$ de los pacientes; a $21,7 \%$ se le realizó una coronariografía y a 1 paciente se le realizó un test de esfuerzo. Posterior a esto fueron revascularizados $6 \%$ del total, con angioplastia 3,6\% y $2,4 \%$ recibió tratamiento quirúrgico.

El promedio de días de hospitalización fue de 9,9 9 6,6 (1-35). Del total de los pacientes, 28 (33,7\%) fallecieron durante la hospitalización, en promedio a los 4,8 7 días (1-35), 25\% antes del cuarto día. La principal causa de muerte intrahospitalaria correspondió al shock cardiogénico, siendo responsable de $57,1 \%$ de las muertes. La distribución de las causas de muerte se expone en la Figura 1.

Al alta hospitalaria, 96,4\% de los pacientes permanecía en tratamiento con aspirina, 41,8\% con IECA, 32,7\% con betabloqueadores, $27,3 \%$ con antagonistas del calcio y $20 \%$ con estatinas.

Con respecto al seguimiento, del total de pacientes dados de alta vivos (55), al año fallecieron 17 pacientes $(30,9 \%)$ y a los 5 años habían fallecido otros 18 pacientes. En total, 35 fallecidos a los 5 años (66,3\%).

Respecto a las causas de muerte extrahospitalaria, 24 pacientes $(68,6 \%)$ correspondieron a causas de tipo cardiovascular. Diez pacientes $(28,6 \%)$ fallecieron por causas no cardiovasculares, y en un paciente no estuvo disponible el documento. Las causas específicas de muerte cardiovascular se detallan en la Tabla 5. Con respecto a la mortalidad no cardiovascular en $80 \%$ (8P) correspondió a neumonía.

\section{DisCUSIÓN}

Del total de pacientes admitidos en nuestro hospital con diagnóstico de IAM, 7\% tenía 80 o más años, cifra superior a la encontrada a nivel poblacional $^{1}$ y similar a la del registro GEMI ${ }^{19}$. Estudios extranjeros en pacientes AM exhiben cifras de hasta $20 \%$ de pacientes mayores de 85 años, mostrando una alta representatividad a nivel mundial ${ }^{8,9}$.

Las características de la población en estudio no difieren en gran medida con lo observado en estudios similares ${ }^{6-8}$, hipertensión arterial y diabetes son los factores de riesgo encontrados en mayor frecuencia ${ }^{6-8}$, ambos estrechamente ligados con la edad, siendo junto a ésta factores de riesgo cardiovascular mayor.

\section{Tabla 5. C ausa de muertes cardiovasculares} durante el seguimiento

\begin{tabular}{|lrc|}
\hline Causa de muerte & N & \% del total \\
\hline Muerte súbita & 11 & 31 \\
Reinfartos & 5 & 14 \\
Insuficiencia cardíaca & 4 & 11 \\
AVE & 3 & 5 \\
TEP & 1 & 3 \\
\hline
\end{tabular}

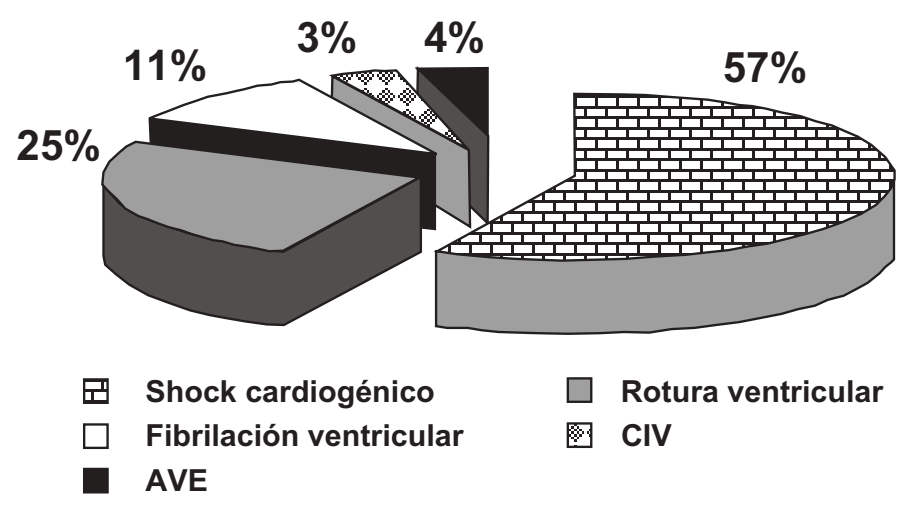

Figura 1. Causas de mortalidad intrahospitalaria por infarto agudo al miocardio. 
Estudios internacionales muestran un alto número de eventos cardiovasculares previos y procedimientos intervencionales tales como angioplastia y cirugía cardíaca ${ }^{7,10}$. En nuestra serie los valores son menores, pudiendo explicarse por las diferencias en las características demográficas de la población, así como también en los sistemas de salud, con recursos disímiles. La mortalidad por IAM en Chile ha disminuido desde la década 1990-9912. Además ha aumentado el acceso a terapias de reperfusión, en especial desde la implementación del plan AUGE, el cual garantiza la utilización de trombolisis en los pacientes que corresponda su uso ${ }^{20}$. La población ha experimentado cambios, aumentado el grupo de AM, todo esto podría determinar cambios sustanciales en el futuro, con un posible aumento de los procedimientos intervencionales, acercándonos a los datos internacionales.

El 78\% de los pacientes consulta previo a las $12 \mathrm{~h}$ de iniciado el cuadro, el porcentaje de pacientes que lo hace antes de las $6 \mathrm{~h}$ disminuye a 59\%. Registros en el país muestran porcentajes similares ${ }^{19}$, existiendo diferencias porcentuales con estudios como el de Sheifer ${ }^{5}$, el cual exhibe cifras del alrededor de $70 \%$ a las $6 \mathrm{~h}$. Pensamos que esto puede basarse en diferencias culturales, económicas y en el conocimiento de la patología coronaria por la población. Sheifer encuentra que la edad (5 años de incremento) es un factor de riesgo independiente de retraso en tiempo de consulta, así como también comorbilidades, como diabetes y otras ${ }^{5}$.

La hemodinamia al ingreso, así como los valores enzimáticos, no difieren con la de otros estudios 7,10 .

Las características electrocardiográficas observadas difieren a las observadas en otros estu$\operatorname{dios}^{10,19}$ en pacientes AM, en que es mayor el porcentaje de IAM sin SDST, primando en el nuestro el SDST, en un grado menor a la población general con IAM descrita por el grupo $\mathrm{GEM}^{22}$. Estas diferencias no tienen una explicación clara, pero un hecho a considerar es que nuestros pacientes tienen un porcentaje significativamente menor de eventos e intervenciones previas, para muchos de ellos éste era su primer evento vascular grave y esto podría ser parte de la explicación de estas diferencias.

Masoudi et $\mathrm{al}^{6}$, estudiaron una cohorte de 20.550 pacientes mayores de 65 años ingresados con IAM, describiendo sus características clínicas y de tratamiento. Con respecto al uso de $ß$ bloqueadores en las primeras $24 \mathrm{~h}$ de evolución, encontraron sólo a $21 \%$ del total de pacientes, cifra similar a la encontrada en el estudio GRACE $^{10}$ y supera los valores encontrados en nuestra serie de 10,8\%, lo que demuestra un sesgo mayor en la prescripción de $\beta$ bloqueo en este grupo etario. Esto podría deberse a una falta de conocimiento de las características propias del IAM en octogenarios, lo que llevaría a conductas más conservadoras, junto con el miedo de complicaciones mayores con uso de estos fármacos en este grupo etario.

La terapia inicial fue en su mayoría farmacológica, existiendo sólo 19\% de pacientes que recibe terapia de reperfusión. Esta cifra es inferior a la encontrada en estudios extranjeros ${ }^{6-8}$, a pesar del beneficio de una terapia agresiva e intervencional precoz en el grupo de octogenarios ${ }^{13,18,22}$. Con el cambio en la ley de salud comentado es de esperar que aumente el número de pacientes sometidos a terapia de reperfusión en el país. Esperamos además que estos trabajos contribuyan a lograr aumentar estas terapias también en este grupo especial de pacientes, en los cuales se ha probado la utilidad de ambas, especialmente de angioplastia primaria ${ }^{23,24}$.

En la evolución hospitalaria, un número importante de pacientes evolucionó con complicaciones, aun así, el número de pacientes sometidos a estudios invasivos es muy reducido, con lo que sólo $6 \%$ de ellos fueron finalmente revascularizados, cifra inferior a la encontrada en otros estudios $^{7}$. Un hecho a considerar aquí es la sensación de temor existente frente a estos pacientes ante procedimientos invasivos, existiendo la creencia de que presentan riesgos altos, estudios del exterior $\mathrm{y}$, recientemente, un estudio en nuestro país desmitifica estas ideas ${ }^{26,27}$, mostrando su factibilidad y bajo riesgo. Esperamos que estos estudios aporten para un cambio de conducta frente a estos pacientes y así mejorar los resultados finales y a largo plazo.

La mortalidad intrahospitalaria exhibida de $33,7 \%$, muy superior a la encontrada en otros estudios $^{6,10}$. La explicación más probable para esta altísima mortalidad en nuestro centro $y$, probablemente en otros del país, es la falta de utilización de tratamientos demostrados a toda edad, como son las terapias de reperfusión y la 
utilización de $ß$ bloqueadores. Junto a esto, destaca el bajo porcentaje de pacientes revascularizados, como vimos antes, muy inferior a estudios extranjeros ${ }^{6-8}$.

El tratamiento farmacológico al alta también destaca por la baja utilización de fármacos probadamente útiles como son las estatinas, beta bloqueadores e inhibidores de la enzima convertidora. Con respecto a los beta bloqueadores, existen estudios demostrados de su beneficio en el uso de éstos en pacientes $\mathrm{AM}^{28}$.

El seguimiento mantiene la tendencia hospitalaria de una alta mortalidad. Estos valores pueden explicarse por las siguientes variables, primero la edad, ya que a mayor edad la expectativa de vida disminuye, siendo más probable morir. Sin embargo, creemos que el manejo intrahospitalario poco agresivo, como se analiza con anterionidad, sella el pronóstico de mortalidad a corto, mediano y largo plazo. Hecho que se afirma en la alta tasa de mortalidad de causa cardiovascular encontrada en nuestra serie (69\%).

Un factor importante que debe considerarse en la interpretación de estos resultados es el tiempo en que se llevó a cabo este registro, que se inició

\section{REFERENCIAS}

1. Instituto Nacional de Estadísticas (INE). Censo 2002 Síntesis de resultados Chile, Santiago 2002. 50 p. Disponible en: www.ine.cl [Consultado 1 de marzo 2007].

2. Chile. Estimaciones y Proyecciones de Población por Sexo y Edad. Total País: 1950-2050, LC/DEM/ R.217, Serie OI № 97, INE-CELADE. Santiago de Chile, 1995. Disponible en: www ine.cl [Consultado 1 de marzo 2007].

3. Krumholz HM. Ischemic heart disease in the elderly. Conferencia especial en el 36 Congreso Nacional de la Sociedad Española de Cardiología. Rev Esp Cardiol 2001; 54: 819-26.

4. Aronow WS. Silent MI. Prevalence and prognosis in older patients diagnosed by routine electrocardiograms. Geriatrics 2003; 58: 24-6, 36-8, 40.

5. Sheifer S, Rathore S, Gersh B, Weinfurt K, Oetgen W, BREALL J ET AL. Time to presentation with acute myocardial infarction in the elderly: Associations with race, sex, and socioeconomic characteristics. Circulation 2000; 102: 1651-6. en 1988 y continuó hasta 2001, algunos de los tratamientos recientemente comentados, que fueron poco usados en este grupo de pacientes, han alcanzado su mayor validez en los últimos años.

Finalmente, podemos concluir que los octogenarios son un grupo cada vez más numeroso, presentan un alto porcentaje de eventos cardiovasculares, especialmente IAM, con un alto índice de complicaciones y mortalidad tanto intrahospitalaria como alejada. Sin embargo, son un grupo insuficientemente estudiado y pobremente tratado, tanto en la fase inicial del cuadro agudo, como en su evolución y al alta. Junto a esto, se agrega el alto riesgo que agrega la edad, condicionándoles un mal pronóstico. Creemos que esto puede cambiar si existe un proceder más activo y con menos sesgos por parte del equipo médico para con estos pacientes, reconociendo precozmente el diagnóstico de IAM y brindando un tratamiento agresivo y precoz de revascularización, luego un mejor manejo farmacológico, estudio invasivo y revascularización a quienes lo requieran. Con esto esperamos mejorar tanto la sobrevida como la calidad de vida de este grupo de pacientes.

6. Masoudi FA, Foody JM, Havranek EP, Wang Y, RADFORD MJ, AшMAN RM ET AL. Trends in acute myocardial infarction in 4 US states between 1992 and 2001: clinical characteristics, quality of care, and outcomes. Circulation 2006; 114: 2806-14.

7. Ko DT, Krumholz HM, Wang Y, Foody JM, Masoudi FA, HAVRANEK EP ET AL. Regional differences in process of care and outcomes for older acute myocardial infarction patients in the United States and Ontario, Canada. Circulation 2007; 115: 196-203.

8. Guaglum G, Stone GW, Cox DA, Stuckey T, Tcheng JE, TURCo M ET aL. Outcome in elderly patients undergoing primary coronary intervention for acute myocardial infarction: results from the Controlled Abciximab and Device Investigation to Lower Late Angioplasty Complications (CADILAC) trial. Circulation 2004; 110: 1598-604.

9. Cequier a, Bueno H, Auge JM, Bardaji A, FernánDEZ-Ortiz A, Heras M. Características y mortalidad después de intervención coronaria percutánea primaria en Infarto Agudo al Miocardio en España. Resultados del registro TRIANA 1 (Tratamiento del Infarto Agudo de Miocardio en Ancianos). Rev Esp Cardiol 2005; 58: 341-50. 
10. Avezum A, Makdisse M, Spencer F, Gore JM, Fox KA, MonTALESCOT G ET AL. GRACE Investigators. Impact of age on management and outcome of acute coronary syndrome: observations from the Global Registry of Acute Coronary Events (GRACE). Am Heart J 2005; 149: 67-73.

11. Thom T, Haase N, Rosamond W, Howard V, Rumsfeld J, Manolio T ET AL. Heart Disease and Stroke Statistics 2006 Update: A Report from the American Heart Association Statistics Committee and Stroke Statistics Subcommittee. Circulation 2006; 113: e85-e151.

12. Szot J. Mortalidad por infarto agudo al miocardio en Chile: 1990-2001. Rev Méd Chile 2004; 132: 1227-33.

13. Peterson ED, Alexander KP, Malenka DJ, Hannan EL, O’Conner GT, McCaLister BD et aL. Multicenter experience in revascularization of very elderly patients. Am Heart J 2004; 148: 486-92.

14. Berger AK, Radford MJ, Wang Y, Krumholz HM. Thrombolytic therapy in older patients. J Am Coll Cardiol 2000; 36: 366-74.

15. Bosch X, SAnchis J. Tratamiento de reperfusión en pacientes de más de 75 años con infarto de miocardio. ¿Necesitamos un estudio controlado y aleatorizado? Rev Esp Cardiol 2005; 58: 333-7.

16. Berger AK, Radford MJ, Krumholz HM. Factors associated with delay in reperfusion therapy in elderly patients with acute myocardial infarction: analysis of the cooperative cardiovascular project. Am Heart J 2000; 139: 985-92.

17. Stenestrand U, WaLientin L, Register of Information and Knowledge About Swedish Heart Intensive Care Admissions (RIKS-HIA). Fibrinolytic therapy in patients 75 years and older with ST-segmentelevation myocardial infarction: one-year followup of a large prospective cohort. Arch Intern Med 2003; 163: 965-71.

18. Graham MM, Ghali WA, Faris PD, Galbraith PD, Norris CM, Knudtson M et al, Alberta Provincial Project for Outcomes Assessment in Coronary Heart DiseASE (APPROACH) INVESTigators. Survival after coronary revascularization in the elderly. Circulation 2002; 105: 2378-84.
19. Prieto JC, Corvalán R, Chávez E, Lanas F, Cumsilie G, Nazzal C. Infarto agudo del miocardio en hospitales chilenos. Resultados finales del estudio GEMI. Rev Méd Chile 1999; 127: 763-74.

20. Ministerio de Salud. Guía Clínica Infarto Agudo del Miocardio y Manejo del Dolor Torácico en Unidades de Emergencia. $1^{\text {st }}$ Ed. Santiago: Minsal, 2005.

21. Prieto J, Corvalán R, Bartolucci J, Nazzal C, Chávez E, LANAS F ET AL. Cambios en el manejo intrahospitalario del infarto del miocardio en hospitales chilenos. Rev Chil Cardiol 2003; 22: 31-6.

22. Tang EW, Wong CK, Herbison P. Global Registry of Acute Coronary Events (GRACE) hospital discharge risk score accurately predicts long-term mortality post acute coronary syndrome. Am Heart J 2007; 153: 29-35.

23. De Boer MJ, Ottervanger JP, van't Hof AW, Hoorntje JC, Suryapranata H, Zijlstra F, Zwolle Myocardial Infarction Study Group. Reperfusion therapy in elderly patients with acute myocardial infarction: a randomized comparison of primary angioplasty and thrombolytic therapy. J Am Coll Cardiol 2002; 39: 1723-8.

24. KeEIEY EC, Boura JA, GRINes CL Primary angioplasty versus intravenous thrombolytic therapy for acute myocardial infarction: a quantitative review of 23 randomized trials. Lancet 2003; 361: 13-20.

25. Granger CB, Goldberg RJ, Dabbous O, Pieper KS, EAgLe KA, CANNon CP ET aL. Predictors of hospital mortality in the global registry of acute coronary events. Arch Intern Med 2003; 163: 2345-53.

26. Ricou FJ, Suilen C, Rothmeier C, Gisselbaek A, Urban P. Coronary angiography in octogenarians: results and implications for revascularization. Am J Med 1995; 99: 16-21.

27. Ugaide H, Dreyse X, Espinosa P, Pizarro G. Angiografía coronaria en pacientes de 80 y más años. Experiencia de 12 años. Rev Chil Cardiol 2007; 26: 29-33.

28. Krumholz HM, Radford MJ, Wang Y, Chen J, Heiat A, MARCINIAK TA. National use and effectiveness of beta-blockers for the treatment of elderly patients after acute myocardial infarction: National Cooperative Cardiovascular Project. JAMA 1998; 280: 623-9. 